\title{
Therapeutic Hydrocortisone
}

National Cancer Institute

\section{Source}

National Cancer Institute. Therapeutic Hydrocortisone. NCI Thesaurus. Code C555.

A synthetic or semisynthetic analog of natural hydrocortisone hormone produced by the adrenal glands with primary glucocorticoid and minor mineralocorticoid effects. As a glucocorticoid receptor agonist, hydrocortisone promotes protein catabolism, gluconeogenesis, capillary wall stability, renal excretion of calcium, and suppresses immune and inflammatory responses. ( $\mathrm{NCl04)}$ 